\title{
Kinetics and subcellular localization of 5-ALA-induced PpIX in DHL cells via two-photon excitation fluorescence microscopy
}

\author{
RONG CHEN ${ }^{1,2}$, ZUFANG HUANG ${ }^{1}$, GUANNAN CHEN ${ }^{1}$, YONGZENG LI ${ }^{1}$, \\ XIANLIAN $\mathrm{CHEN}^{3}$, JIANXIN $\mathrm{CHEN}^{1}$ and HAISHAN ZENG ${ }^{2}$
}

\begin{abstract}
${ }^{1}$ Key Laboratory of Optoelectronic Science and Technology for Medicine, Ministry of Education, Fujian Normal University, Fuzhou, Fujian 350007, P.R. China; ${ }^{2}$ Cancer Imaging Department, British Columbia Cancer Research Centre, 675 West 10th Avenue, Vancouver, BC, V5Z 1L3, Canada; ${ }^{3}$ Fujian Institute of Hematology, Union Hospital, Fujian Medical University, Fuzhou, Fujian 350001, P.R. China
\end{abstract}

Received October 24, 2007; Accepted December 28, 2007

\begin{abstract}
Two-photon excitation fluorescence (TPEF) microscopy was used to measure the 5-aminolevulinic acid (5ALA)-induced PpIX fluorescence in follicular lymphoma DHL cells. Kinetics of 5-ALA-induced PpIX accumulation in DHL cells under various 5-ALA concentrations was studied. We found that during the course of continuous incubation with 5-ALA, the relationship between the DHL cell fluorescence signal and the incubation time showed a biphasic variation. Initially the PpIX signal increased with the incubation time and reached the maximal value at about $3 \mathrm{~h}$, and then it decreased with time during the subsequent incubation period. By labeling the 5-ALA incubated DHL cells with different organelle-specific fluorescence probes: Rhodamine 123 (for mitochondria), $\operatorname{DioC}_{6}(3)$ (for endoplasmic reticulum) and LysoTracker Green (for lysosomes) respectively, we found that 5-ALA-induced PpIX was primarily localized in endoplasmic reticulum and mitochondria; its concentration in the lysosome was much lower. The results suggested that 5-ALA could potentially be an effective photosensitizer in photodynamic purging of DHL cells. Two-photon excitation fluorescence microscope is a useful tool for studying 5-ALA-induced PpIX subcellular localization.
\end{abstract}

Correspondence to: Dr Haishan Zeng, Cancer Imaging Department, British Columbia Cancer Research Centre, 675 West 10th Avenue, Vancouver, BC, V5Z 1L3, Canada

E-mail: hzeng@bccrc.ca

Abbreviations: 5-ALA, 5-aminolevulinic acid; PpIX, protoporphyrin IX; TPEF, two-photon excitation fluorescence; LSM, laser scanning microscope; PDT, photodynamic therapy

Key words: two-photon excitation fluorescence, 5-aminolevulinic acid, protoporphyrin IX, photosensitizer subcellular localization, DHL cells, photodynamic therapy

\section{Introduction}

In recent years, 5-aminolevulinic acid (5-ALA) has been used in photodynamic diagnose and photodynamic therapy (PDT) studies of various cell lines and diseases including pre-cancer (actinic keratosis) and invasive cancers (1-4), while PDT purging of leukemia cells has become a subject of intensive research (5-7). 5-ALA is the precursor of protoporphyrin IX (PpIX), when it is administered to patients, PpIX is produced through the heme biosynthetic pathway. Because of the significant difference in the activities of key enzymes in the heme pathway between tumor and normal tissue, the PpIX accumulation in tumor cells is generally higher than that in normal cells $(8,9)$. PpIX can lead to photosensitization by a broad spectrum of light especially when it is irradiated by blue light around $410 \mathrm{~nm}$ or by red light around $630 \mathrm{~nm}$ (10).

5-ALA has been successfully used in the photodynamic purging of HL60 cells, K562 cells and other leukemia cells (11-14). However, to the best of our knowledge, there is no previous report on the photodynamic purging of lymphoma cells. There are many different types of lymphoma. Our interest is to use DHL cells available in our laboratory as an example to study PDT purging for lymphoma therapy. DHL cells are a follicular lymphoma cell line. The objectives of this work were to study the sensitization of DHL cells to 5ALA, the kinetics of 5-ALA-induced PpIX in DHL cells; and the localization of PpIX within DHL cells. Previous studies have demonstrated that the pattern of photosensitizer subcellular localization correlated with the photodynamic mechanisms and photodynamic efficiency (15-18). Therefore, this study is very important for developing an optimized procedure for photodynamic purging of DHL cells using 5ALA as the photosensitizer.

The kinetics of 5-ALA induced PpIX in leukemia cells has been studied previously using macro-spectrofluorometer measurements of cell samples in cuvette (19). In these types of experiments, 5-ALA was first incubated with leukemia cells for different time periods. Then the incubated cells were centrifuged to remove the debris. The characteristic fluorescence of PpIX in the cell samples was then measured in a spectrofluorometer and the concentration of PpIX was 
derived by comparing with PpIX fluorescence calibration standard. This experimental procedure was complicated and the results reflected only the average PpIX concentrations in the sample, not the real PpIX concentration in single cells. In this study, we used two-photon excitation fluorescence microscopy to study the kinetics of 5-ALA induced PpIX in DHL cells. In combination with organelle-specific optical probes, subcellular distribution and localization of 5-ALAinduced PpIX were also investigated in this work. Different from conventional one-photon excitation fluorescence measurement methods, two-photon fluorescence microscopy uses femtosecond laser as the excitation source and the fluorescence excitation takes place only within the focus region of the laser beam. Although the instantaneous intensity is high, the average power reached the sample is low and much less photobleaching and/or photodamaging occurs in the measured biological samples (20-23).

\section{Materials and methods}

Chemicals and cell culture. 5-Aminolevulinic acid (5-ALA) and RPMI-1640 were purchased from Sigma. 5-ALA was dissolved in RPMI-1640 (serum-free), filter-sterilized and stored at $4^{\circ} \mathrm{C}$ before use. Three different organelle-specific fluorescence probes: Rhodamine 123 for mitochondria, $\mathrm{DioC}_{6}(3)$ for endoplasmic reticulum, and LysoTracker Green for lysosomes were purchased from Molecular Probes Inc. All other chemicals used were of analytical reagent grade.

DHL cells (follicular lymphoma) were kindly provided by Shanghai Institute of Hematology and were cultured in RPMI-1640 medium supplemented with $10 \%$ fetal calf serum (FCS), $100 \mathrm{U} / \mathrm{ml}$ penicillin, and $50 \mathrm{mg} / \mathrm{ml}$ streptomycin (Gibco, Invitrogen) in a humidified atmosphere with $5 \% \mathrm{CO}_{2}$ and at $37^{\circ} \mathrm{C}$. The cells were subcultured 3 times every week to keep the exponential growth.

Measurement of cellular accumulation of 5-ALA-induced PPIX. 5-ALA solution was added into the culture medium with the final concentrations of 2,4 and $10 \mathrm{mmol} / \mathrm{l}$, and the incubation time was varied from 0 to $7 \mathrm{~h}$. The cell density in the cell-ALA mixtures is in the order of $10^{6}$ cells $/ \mathrm{ml}$. The cells were washed twice with iced PBS and resuspended in fresh medium. A drop of the mixture ( $\sim 1000$ cells) were put onto the microscope slide for imaging investigation. Following different incubation time, PpIX content in DHL cells was evaluated using the Zeiss LSM 510 META laser scanning microscope equipped with a mode-locked femto-second Ti: sapphire laser (110 fs, $76 \mathrm{MHz}$ ), tunable from 700 to $980 \mathrm{~nm}$ (Coherent Mira 900-F, USA), as shown in Fig. 1. The excitation wavelength was set at $820 \mathrm{~nm}$, while the fluorescence emission from the DHL cells was directed by the main dichroic beam splitters (MDBS) to the META detector. The META detector with 32-gated photon counting module was used to collect images at a series of emission wavelengths range from 377 to $716 \mathrm{~nm}$. In Lambda mode, analyzed cells were captured using $512 \times 512$ pixel measuring window with a scanning speed of 8 corresponding to a total scanning time of $1.57 \mathrm{sec}$, and the average time per pixel was $2.56 \mu \mathrm{sec}$. Fluorescence signal from single DHL cells was measured in the spectral range from 586 to $714 \mathrm{~nm}$ by the META detector. The system software allows the user to select any region of interest for spectral measurements. When measuring a particular cell, we first found the largest section of that cell, and then defined the whole cell section as the region of interest for spectral analysis. An example spectrum from a region of interest defined by the circle contour in Fig. 2A is shown in Fig. 2B. Twenty randomly selected individual cells in each sample corresponding to a particular 5-ALA incubation time were analyzed under the same system parameters. The average PpIX fluorescence peak intensity from the 20 cells was considered to represent the cellular PpIX level of the measured cell sample corresponding to a specific incubation time. We expect that the consistent sampling procedure and the averaging over 20 cells will minimize the error caused by heterogeneous PpIX distribution in individual cells.

Subcellular localization of ALA-induced PpIX in DHL cells. 5-ALA and organelle-specific probes were prepared with parameters as shown in Table I. The organelle-specific probes (Molecular Probes, Eugene, OR) included probes specific to mitochondria (Rhodamine 123), lysosomes (LysoTracker Green) and endoplasmic reticulum apparatus $\left[\mathrm{DioC}_{6}(3)\right]$. DHL cells were incubated with 5-ALA for $\sim 3 \mathrm{~h}$, then centrifuged to obtain a cell pellet, aspirated the supernatant, and resuspended the cells gently in probe-containing medium. Then the cells were incubated for another $0.5 \mathrm{~h}$ in the $\mathrm{CO}_{2}$ incubator with one of the three organelle-specific probes. Finally the cells were washed twice with iced PBS and resuspended in fresh medium before imaging investigation.

The same microscope system shown in Fig. 1 is used for both the 2-photon excitation fluorescence study using the fs laser and the conventional one photon fluorescence analysis using a-488 nm CW Argon-ion laser. The organelle-specific probes were excited by the 488-nm CW laser light, while 5-ALA-induced PpIX was excited by the $820 \mathrm{~nm}$ fs pulses from the Ti:Sapphire laser and a KP685 filter (685 nm short pass) was used for acquiring the fluorescence image. A Plan-Apochromat 63 x (N.A. = 1.4) oil immersion objective (Zeiss) was employed for focusing the excitation beam and collecting the backward fluorescence signals. META detector which covers a spectral range from 377 to $716 \mathrm{~nm}$ was used to record the emission signals. In the experiment, we used two independent channels, so that they can acquire images with best contrast under the optimal system parameters, including the laser intensity, detector gain, amplifier offset. In order to avoid the emission fluorescence crosstalk between fluorescent probes and ALA-induced PpIX, only one of the three different organelle-specific probes was added into DHL cells incubated with 5-ALA in a given experimental sample. As shown in Table I, emission filters were properly chosen for different organelle-specific probes according to the spectral features obtained from Invitrogen Inc. Additionally, in the independent channel, META detector which colleted the emission fluorescence of PpIX was set to a spectral range from 585 to $714 \mathrm{~nm}$.

\section{Results and Discussion}

Kinetics of ALA-induced PpIX in DHL cells. Fluorescence is an indirect method for quantifying photosensitizer (including 


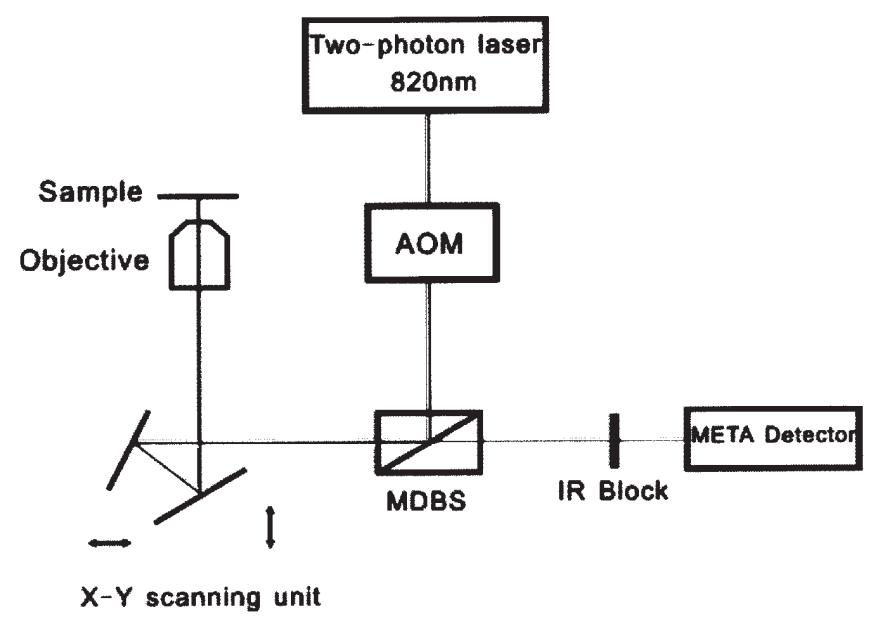

Figure 1. Schematics of the Zeiss LSM 510 META system for two-photon excitation fluorescence imaging and spectral analysis. AOM, acousto-optic modulator; MDBS, main dichroic beam splitter; IR block, filter for blocking the excitation light.

PpIX) distributions and localization in tissue and cells. Its drawbacks and advantages have been well documented in literature with studies of PpIX and a variety of other fluorescing photosensitizers. Many published studies compared the measurement fluorescence signals in situ/in vivo with HPLC measurements of tissue/cell extraction (24-26). These studies were performed with conventional one-photon excitation fluorescence technique. In this study, we used two-photon excitation fluorescence microscope for in situ determination of the relative PpIX content changes and localization in DHL cells. We recognize that TPEF will inherit all the advantages and drawbacks of one photon fluorescence technique, but will bring additional advantages in confocal microscopic analyses of tissues and cells. These advantages are less photobleaching and less photodamage to the sample due to the fact that two-photon fluorescence excitation occurs only at the focal point, not the whole beam path and that less average laser power is required than for conventional fluorescence confocal measurement because of the elimination of the detection pinhole in two-photon excitation fluorescence measurement, therefore, more fluorescence photons being collected. These advantages have been well recognized by researchers studying biological samples (20-23). The disadvantages of two-photon excitation fluorescence are more expensive equipment and the incapability of performing large volume sampling at one time because for two-photon fluorescence excitation to occur, the fs laser beam has to be focused to a micro-spot to achieve instantaneously high light intensity. In our experiments, PpIX content was evaluated using the LSM software. It is proportional to the average fluorescence intensity per cross-section area of the analyzed cells. In each sample, we analyzed 20 randomly selected individual cells to generate statistically meaningful data.

At all tested 5-ALA concentrations (2, 4 and $10 \mathrm{mmol} / \mathrm{l})$, continuous incubation of DHL cells resulted in significant PpIX accumulation in the cells as evident from the observed PpIX characteristic fluorescence signals. Results of PpIXspecific fluorescence intensity estimated from the TPEF microscopy analysis are presented in Fig. 3. At all ALA concentrations, the relationship between the PpIX fluorescence in DHL cells and the incubation duration showed biphasic characteristics. Initially, the PpIX fluorescence signal in DHL cells increased with increasing incubation time and reached maximum at $3 \mathrm{~h}$ : 1310 a.u. for $2 \mathrm{mmol} / 1$ 5-ALA concentration, 1562 a.u. for $4 \mathrm{mmol} / \mathrm{l}$ 5-ALA. However, for $10 \mathrm{mmol} / \mathrm{l}$ 5-ALA concentration, the maximum fluorescence intensity decreased to 1278 a.u. and it occurred at 4-h incubation time. It is fair to say that $3 \mathrm{~h}$ of 5-ALA incubation is about the optimal incubation time for PpIX accumulation in DHL cells.

5-ALA is a precursor of porphyrin in the biosynthetic pathway of heme. 5-aminolevulinate synthase catalyzes the conversion of glycine and succinyl coenzyme A (CoA) into 5-aminolevulinic acid molecules. 5-ALA is finally transformed into PpIX in the mitochondrial $(6,8)$. Normally heme inhibits the formation of endogenous PpIX by a negative feedback control mechanisms, however, exogenous 5-ALA
(A)

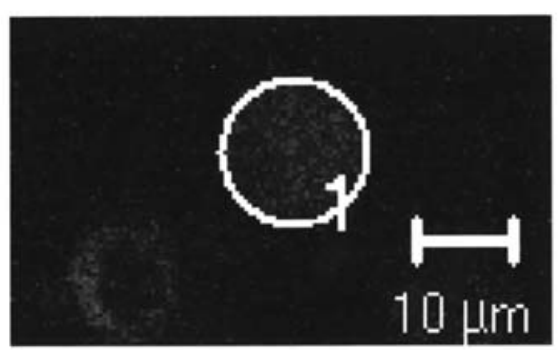

(B)

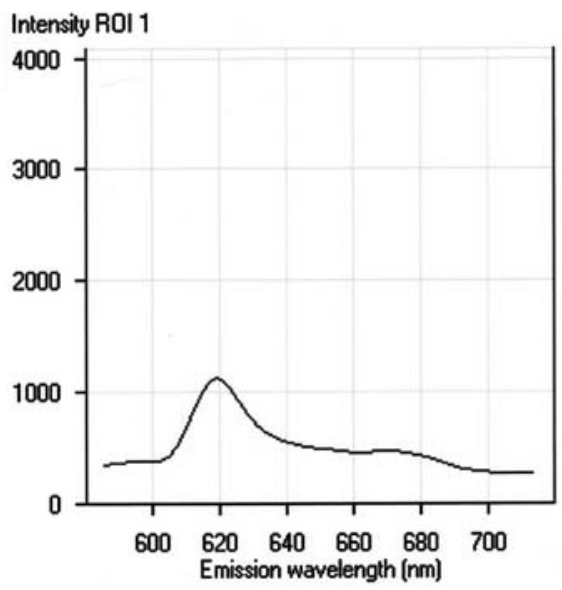

Figure 2. In Lambda mode, corresponding fluorescence spectrum (B) excited by $820 \mathrm{~nm}$ was obtained from the region of interest (white circle) in a DHL cell (A). 
Table I. Preparation of DHL cells - concentrations of 5-ALA and organelle-specific probes in the cell culture medium, incubation time, excitation wavelength, and the filters for imaging.

\begin{tabular}{lcccc}
\hline Photosensitizer/probe & Concentration in medium & Incubation time & Excitation wavelength & Imaging filters \\
\hline 5-ALA & $4 \mathrm{mmol} / 1$ & $3 \mathrm{~h}$ & $820 \mathrm{~nm}$ (2-photon) & KP685 \\
$\begin{array}{l}\text { Rhodamine } 123 \\
\text { (mitochondrial probe) }\end{array}$ & $10 \mu \mathrm{g} / \mathrm{ml}$ & $0.5 \mathrm{~h}$ & $488 \mathrm{~nm}$ & BP505-550 \\
$\begin{array}{l}\text { LysoTracker Green } \\
\text { (lysosomal probe) }\end{array}$ & $100 \mathrm{nM}$ & $0.5 \mathrm{~h}$ & $488 \mathrm{~nm}$ & BP505-530 \\
$\begin{array}{l}\text { DioC } 6 \text { (3) (endoplasmic } \\
\text { reticulum probe) }\end{array}$ & $2 \mu \mathrm{g} / \mathrm{ml}$ & $0.5 \mathrm{~h}$ & $488 \mathrm{~nm}$ & BP505-530 \\
\hline
\end{tabular}
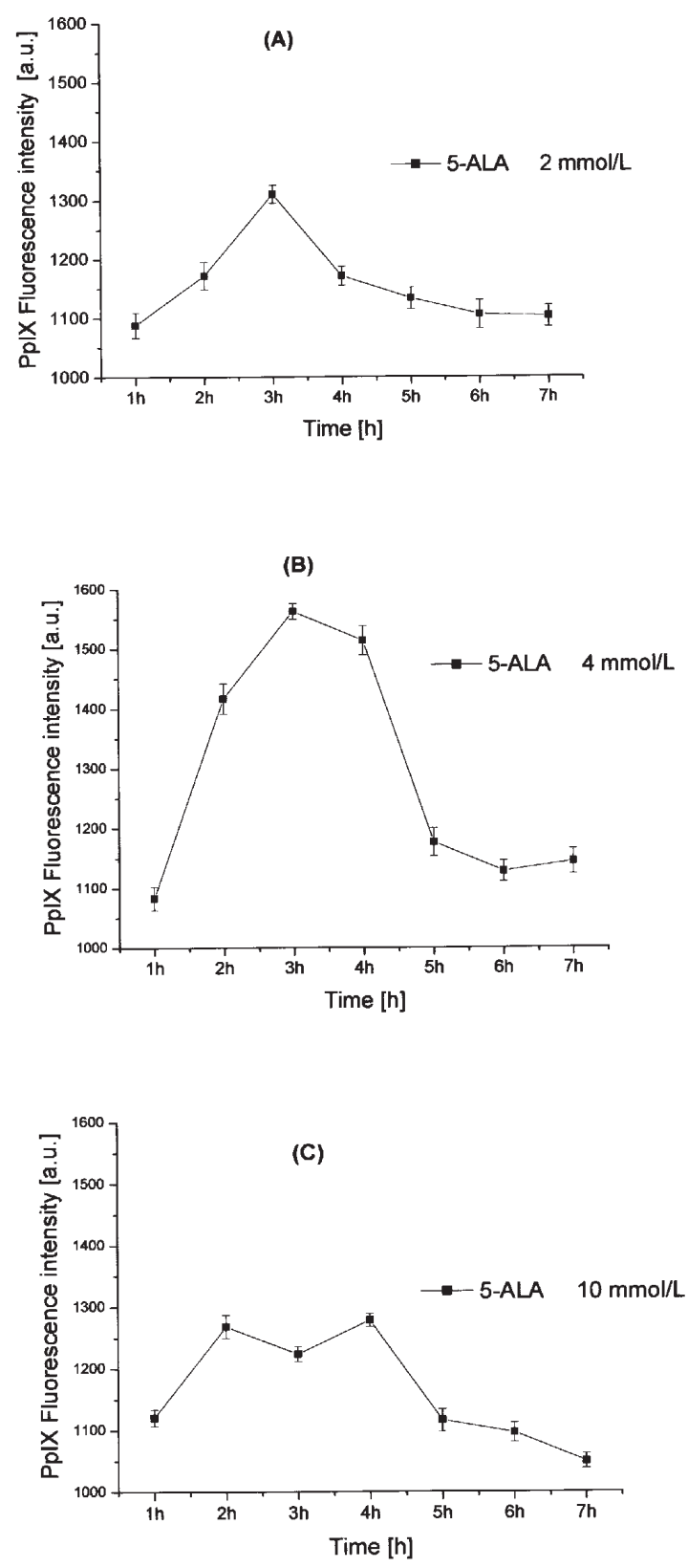

Figure 3. Accumulation of PpIX in DHL cells during continuous incubation with 5-ALA. Error bars represent calculated standard deviations. (A) Incubated with $2 \mathrm{mmol} / \mathrm{l}$ of 5-ALA solution. (B) Incubated with $4 \mathrm{mmol} / \mathrm{l}$ of 5-ALA solution. (C) Incubated with $10 \mathrm{mmol} / \mathrm{l}$ of 5-ALA solution. bypasses this regulatory mechanism and results in a temporary high concentration of PpIX accumulation (27). In our experiments, three different concentrations of 5-ALA solution $(2,4$ and $10 \mathrm{mmol} / \mathrm{l})$ were added into the DHL cell culture and incubated for several hours, however, the induced PpIX concentrations were not proportional to 5-ALA concentration. Moreover, higher 5-ALA concentration did not necessarily result in proportionally higher PpIX formation. The results showed that when the 5-ALA concentration was increased to $10 \mathrm{mmol} / \mathrm{l}$, the induced PpIX in DHL cells actually decreased as compared to that for 2 and $4 \mathrm{mmol} / \mathrm{l} \mathrm{5-}$ ALA concentrations. One of the reasons may be that 5-ALA in higher concentration solutions tend to aggregate easier than at lower concentrations (28). Another reason may be that the viability of DHL cells, especially the mitochondria in the cells, was affected when incubated with higher concentrations of 5-ALA. The exact mechanism for this phenomenon deserves further investigations.

Subcellular localization of ALA-induced PpIX. DHL cells incubated with 5-ALA and then stained with one of the three fluorescence probes were studied using the system shown in Fig. 1. PpIX intracellular distribution was imaged by twophoton excitation fluorescence technique under $820 \mathrm{~nm}$ fs laser excitation, while the distribution of the organellespecific fluorescence probe in cells was imaged with conventional fluorescence technique under $488 \mathrm{~nm} \mathrm{CW}$ laser excitation. The results are shown in Fig. 4. PpIX fluorescence is presented in red color in the left column. Rhodamine 123, $\operatorname{DioC}_{6}(3)$ and LysoTracker Green fluorescence probes are presented in green color in the middle column. The overlay regions which appeared in yellow color indicate that PpIX appeared at the same place as the fluorescence probes (right column).

It is well known that photosensitizer localization pattern is an important determinant for its photodynamic efficiency. Studies have shown that different localization sites of photosensitizer in tumor cells correlated with different photodynamic action mechanisms and resulted in different PDT efficiencies (29). Studying the subcellular localization of photosensitizer will help locate the sites inside the cell where photodynamic process taking place and therefore, improves the understanding of PDT mechanisms. Previous studies have demonstrated 


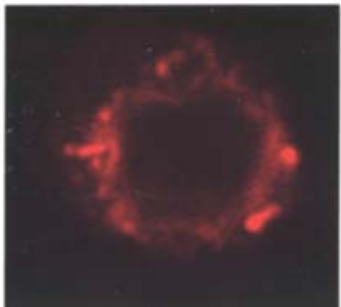

PplX

(A)

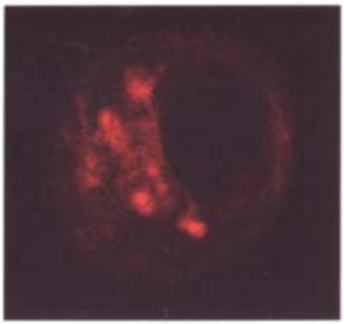

(B)

PplX

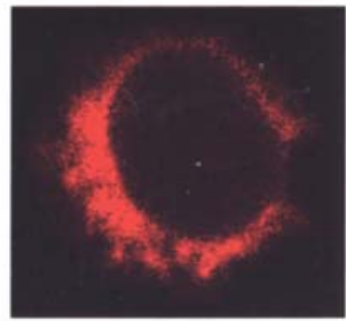

(C)

PpIX

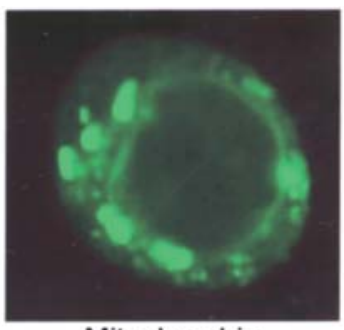

Mitochondria

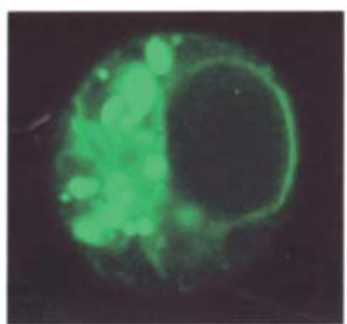

Lysosome

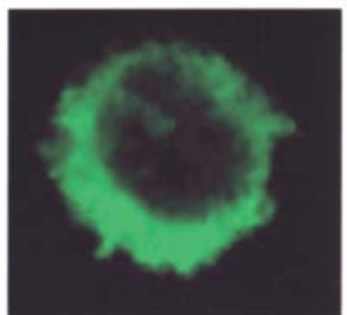

Endoplasmic Reticulum

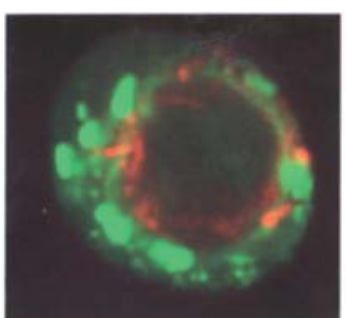

PpIX + Mitochondria

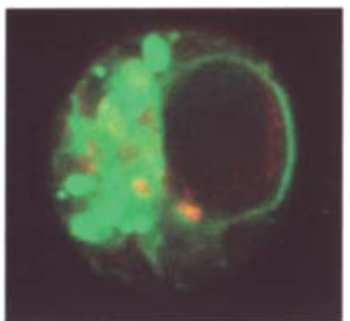

PplX + Lysosome

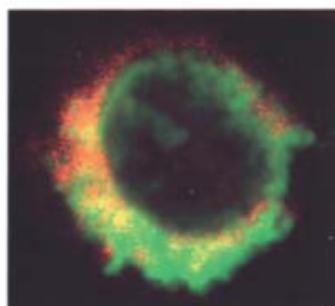

PplX + Endoplasmic Reticulum

Figure 4. Subcellular localization of ALA-induced PpIX in DHL cells. (A) Mitochondria probe images, PpIX is shown in red, the mitochondria probe is shown in green, areas of overlap between two images is shown in yellow. (B) Lysosome probe images, PpIX is shown in red, the lysosome probe is shown in green, areas of overlap between two images is shown in yellow. (C) Endoplasmic reticulum probe images, PpIX is shown in red, the endoplasmic reticulum probe is shown in green, areas of overlap between two images is shown in yellow.

that higher PpIX concentration was induced in mitochondria from exogenous ALA $(8,30)$. Zhen et al found that 5-ALAinduced PpIX was primarily confined to the mitochondria and resulted in better PDT efficiency than that of exogenous PpIX, which was distributed in the whole cytoplasm of cells (15). One should also keep in mind that the primary localization site depends on the photosensitizer type, concentration and the cell lines $(31,32)$. In this study, we used double-labeling (33) and two-photon excitation fluorescence microscopy as well as conventional confocal fluorescence microscopy to determine the localization of 5-ALA-induced PpIX. As shown in Fig. 4, after incubated with 5-ALA for about 3 h, 5-ALA-induced PpIX was primarily localized in endoplasmic reticulum and mitochondria. In addition, ALA-induced PpIX was also localized in lysosome, but seemed to be at much lower concentration than at the other two organelles. Our experiments demonstrated that the distribution of 5-ALA-induced PpIX in cells follows the order: endoplasmic reticulum $>$ mitochondria $>$ lysosome.

It has to be pointed out that PpIX distribution in cells may be dynamic during the 5-ALA incubation process or during light irradiation. We have just studied the subcellular localization of PpIX in DHL cells at the 3-h incubation time-point where the PpIX fluorescence intensity reach its maximum value. More detailed studies of PpIX subcellular localization kinetics may help optimize the PDT treatment effect. Two-photon excitation fluorescence microscopy technique is ideally suitable for such studies due to reduced photobleaching and photodamage. We plan to perform such studies in the future.

In the two-photon excitation fluorescence microscopy experiment, we compared fluorescence images obtained under different excitation wavelengths including 800, 810, 820 and $830 \mathrm{~nm}$. The $820-\mathrm{nm}$ excitation gives the best images. To test whether the excitation of organelle-specific probes and PpIX affected each other, we experimented using 488-nm excitation of 5-ALA-incubated DHL cells in the absence of organelle-specific probes and found that only background noise was detected with no indication of PpIX fluorescence. However, when Rhodamine 123 was added to DHL cells in the absence of 5-ALA, Rhodamine 123 was excited by $810-\mathrm{nm}$ fs lasers. We found that the two-photon excitation fluorescence spectrum of Rhodamin 123 is similar to the single photon excitation fluorescence spectrum measured with 488-nm excitation. This is consistent with the observation of $\mathrm{Xu}$ and Webb that a fluorophore's emission spectra are the same under one-photon excitation and twophoton excitation; and the emission spectral shape are 
independent of excitation wavelengths (34). In order to avoid the influence of 820-nm excitation to Rhodamine 123 and other fluorescence probes, in our experiment organellespecific probes were always excited first during the doublelabel imaging procedures. META detector with a spectral range from 585 to $714 \mathrm{~nm}$ in META channel was independently used for collecting the fluorescence image of PpIX, and a BP filter (500-550 nm) was used for collecting the fluorescence image of Rhodamine 123.

In conclusion, we used two-photon excitation fluorescence microscopy to study the kinetics and subcellular localization of 5-ALA-induced PpIX in DHL cells. DHL cells were incubated with 5-ALA for 0-7 h, two-photon excitation fluorescence microscopy were used to image the PpIX distribution and calculate the relative PpIX content in cells at different incubation time-point. The results demonstrated that 5-ALA incubated with DHL cells can be effectively transferred into PpIX and accumulated in DHL cells. Incubation was performed with three different 5-ALA concentrations, it was found that the induced PpIX in DHL cells reached the maximum value at $\sim 3$-h time-point; as the incubation duration increased further PpIX content accumulated in DHL cells decreased with time. In combination with organelle-specific fluorescence probes, double-label method was used to determine the localization of endogenous PpIX in cells. With the multichannel mode microscope, we used two independent-channels (META channel for PpIX, traditional confocal channel for fluorescence probes) to achieve fluorescence imaging of organelle-specific probes and 5-ALA-induced PpIX, and the overlaid images were presented to find out the subcellular localization of PpIX. The results demonstrated that 5-ALA-induced PpIX were primarily localized in endoplasmic reticulum and mitochondria. In addition, 5-ALA-induced PpIX was also found in lysosome, but at much lower concentrations. Based on the kinetics and subcellular localization of 5-ALA-induced PpIX in DHL cells as revealed in this study, 5-ALA could be used as an effective photosensitizer for photodynamic purging of DHL cells. The study also suggested that twophoton excitation fluorescence microscopy is a useful tool for studying photosensitizer accumulation kinetics and subcellular localization in cells.

\section{Acknowledgements}

The authors wish to thank Peimin Jia, Shanghai Institute of Hematology, for kindly providing the DHL cells. This work was supported by the National Natural Science Foundation of China (International Cooperation Grant no. 60778046 and no. 60711120031) and the Canadian Institutes of Health Research International Scientific Exchange Program.

\section{References}

1. Soler AM, Warloe T and Taujo J: Photodynamic therapy of residual or re-current basal cell carcinoma after radiothemethylester. Acta Oncol 39: 605-609, 2000.

2. Goldman M and Atkin D: ALA/PDT in the treatment of actinic keratosis: spot versus confluent therapy. J Cosmetic Laser Ther 5: 107-110, 2003.

3. Wu RWK, Chu ESM, Yow CMN and Chen JY: Photodynamic effects on nasopharyngeal carcinoma (NPC) cells with 5aminolevulinic acid or its hexylester. Cancer Lett 242: 112-119, 2006.
4. Madsen SJ, Sun CH, Tromberg BJ, Wallace VP and Hirschberg H: Photodynamic therapy of human glioma spheroids using 5-aminolevulinic acid. Photochem Photobiol 72: $128-134,2000$.

5. Kamano H, Okamoto K, Sakata I, Kubota Y and Tanaka T: Photodynamic purging of leukemia cells by high-brightness Light Emitting Diode and gallium-metal porphyrin. Lasers Electro-Optics 3: 1006-1007, 1999.

6. Bartosova J and Hrkal Z: Accumulation of protoporphyrin-IX (PpIX) in leukemia cell lines following induction by 5 -aminolevulinic-acid (ALA). Comp Biochem Physiol Part C 126: 245$252,2000$.

7. Zhang BQ, Miao LX, Zhang ZX, M Xiao and Chen ML: An experimental study on destruction of K562 and HL60 induced by 5 -aminolaevulinic acid-based photodynamic therapy. J Biomed Eng 22: 525-529, 2005.

8. Kennedy JC, Pottier RH and Pross DC: Photodynamic therapy with endogenous protoporphyrin IX: basic principles and present clinical experiences. J Photochem Photobiol B Biol 6: 143-148, 1990.

9. Zenzen V and Zankl H: Protoporphyrin IX-accumulation in human tumor cells following topical ALA- and h-ALAapplication in vivo. Mutat Res 202: 35-42, 2003.

10. Gold MH: 5-Aminolevulinic acid in photodynamic therapy - an exciting future. In: US Dermatol Rev 2006. Tomala L (ed.) Touch Briefings, London pp81-84, 2006.

11. Xiao H, Xiong JW, Wu JM and Zhang ZX: Research of parameters on ALA-PDT destruction of leukemia cell. Acta Laser Biol Sin 13: 353-357, 2004.

12. Wu JM, Xiong JW, Xiao H and Zhang ZX: An experimental research in ALA-PDT by the use of a general white light lamphouse. Laser J 25: 87-88, 2004.

13. Wei L, Wen JZ and Kazunori O: 5-ALA-mediated photodynamic therapy in multidrug resistant leukemia cells, J Photochem Photobiol B Biol 60: 79-86, 2001.

14. Kuzelova K, Grebenova D, Pluskalova M, Marinov I and Hrkal Z: Early apoptotic features of K562 cell death induced by 5 -aminolevulinic acid-based photodynamic therapy. J Photochem Photobiol B Biol 73: 67-78, 2004.

15. Zhen YJ, Yang G, Vasovic V, Cunderlikova B, Suo Z, Nesland JM and Peng Q: Subcellular localization pattern of protoporphyrin IX is an important determinant for its photodynamic efficiency of human carcinoma and normal cell lines. J Photochem Photobiol B Biol 84: 213-220, 2006.

16. Grebenova D, Kuzelova, Smetana K, Pluskalova M, Cajthamlova H, Marinov I, Fuchs O, Soucek J, Jarolim P and Hrkal Z: Mitochondrial and endoplasmic reticulum stressinduced apoptosis pathways are activated by 5 -aminolevulinic acid-based photodynamic therapy in HL60 leukemia cells. J Photochem Photobiol B Biol 69: 71-85, 2003.

17. Oleinick NL and Evans HH: The photobiology of photodynamic therapy: cellular targets and mechanisms. Radiat Res 150 (Suppl.): S146-S156, 1998.

18. Granville DJ and Hunt DWC: Porphyrin-mediated photosensitization taking the apoptosis fast lane. Curr Opin Drug Discov 7: 232-243, 2000.

19. Chen JY, Mak NK, Cheung NH, Leung RN and Peng Q: Endogenous production of protoporphyrin IX induced by 5 aminolevulinic acid in leukemia cells. Acta Pharmacol Sin 22: 163-168, 2001.

20. Denk W, Strickler JH and Webb WW: Two-photon laser scanning fluorescence microscopy. Science 248: 73-76, 1990.

21. Koester HJ, Baur D, Uhl R, and Hell SW: $\mathrm{Ca}^{2+}$ fluorescence imaging with picoand femtosecond two-photon excitation: signal and photodamage. Biophys J 77: 2226-2236, 1999.

22. Hopt A and Neher E: Highly nonlinear photodamage in twophoton fluorescence microscopy. Biophys J 80: 2029-2036, 2001.

23. Rubart M: Two-photon microscopy of cells and tissue. Circ Res 95: 1154-1166, 2004.

24. Zhou S, Zong Y, Nair G, Stewart CF and Sorrentino BP: Increased expression of the Abcg2 transporter during erythroid maturation plays a role in decreasing cellular protoporphyrin IX levels. Blood 105: 2571-2576, 2005.

25. Popken G, Wetterauer U and Schultze-Seemann W: Kidneypreserving tumor resection in renal cell carcinoma with photodynamic detection by 5 -aminolevulinic acid: preclinical and preliminary clinical results. BJU Int 83: 578-582, 1999.

26. Bissonnette R, Zeng H, McLean DI, Schyeiber WE, MacAulay C, Roscoe DL and Lui H: Psoriatic plaques exhibit red autofluorescence that is due to protoporphyrin IX. J Invest Dermatol 111: 586-591, 1998. 
27. Peng Q, Berg K, Moan J, Kongshaug M and Nesland JM: 5aminolevulinic acid-based photodynamic therapy: principles and experimental research. Photochem Photobiol 65: 235-251, 1997.

28. Gadmar ØB, Moan J, Scheie E, Ma LW and Peng Q: The stability of 5-aminolevulinic acid in solution. J Photochem Photobiol B Biol 67: 187-193, 2002.

29. Hang H, Shin DS, Lee YE, Nguyen DC, Trang TC, Pan AH, Huang SLJ, Chong DH and Berns MW: Subcellular phototoxicity of 5-aminolevulinic acid (ALA). Lasers Surg Med 22: 14-24, 1998.

30. Kennedy JC and Pottier RH: Endogenous protoporphyrin IX, a clinically useful photosensitizer for photodynamic therapy. J Photochem Photobiol B Biol 14: 275-292, 1992.

31. Lin CW, Shulok JR, Kirley SD, Cincotta L and Foley JW: Lysosomal localization and mechanism of uptake of nile blue photosensitizers in tumor cells. Cancer Res 51: 2710-2719, 1991.
32. Xue LY, He J and Oleinick NL: Rapid tyrosine phosphorylation of HS1 in the response of mouse lymphoma L5178Y-R cells to photodynamic treatment sensitized by the phthalocyanine Pc4. Photochem Photobiol 66: 105-113, 1997.

33. Wilson BC, Olivo M and Singh G: Subcellular localization of photofrin and aminolevulinic acid and photodynamic crossresistance in vitro in radiation-induced fibrosarcoma cells sensitive or resistant to photofrin-mediated photodynamic therapy. Photochem Photobiol 65: 166-176, 1997.

34. Xu C and Webb WW: Measurement of two-photon excitation cross sections of molecular fluorophores with data from 690 to 1050 nm. J Opt Soc Am B 13: 481-491, 1996. 